\title{
Dynamic behaviors of a local modified stochastic Swift-Hohenberg equation with multiplicative noise
}

\author{
Chunxiao Guo ${ }^{1 *}$, Yanfeng Guo ${ }^{2}$ and Chengyuan $\mathrm{Li}^{1}$
}

\section{"Correspondence:}

guochunxiao1983@sina.com

'Department of Mathematics, China

University of Mining and

Technology Beijing, Beijing, 100083,

China

Full list of author information is

available at the end of the article

\begin{abstract}
In this paper, we investigate a global random attractor for a stochastic local modified Swift-Hohenberg equation with multiplicative noise in Stratonovich sense. Through the Ornstein-Uhlenbeck (O-U) transformation, we obtain the random dynamical system associated with the stochastic local modified Swift-Hohenberg equation. Using the properties of the $\mathrm{O}-\mathrm{U}$ process, we derive the specific uniform a priori estimates, using which we prove the existence of global random attractor for the corresponding random dynamical system.
\end{abstract}

Keywords: local modified stochastic Swift-Hohenberg equation; uniform a priori estimates; random attractor

\section{Introduction}

Swift and Hohenberg [1] proposed a model for the convective instability in the RayleighBénard convection, also known as the Swift-Hohenberg (S-H) equation, which is included as an important equation in different branches of physics, such as Taylor-Couette flow [2, 3], the study of lasers [4], and so on. After that, Doelman and Standstede [5] proposed the following modified Swift-Hohenberg equation for a pattern formation system near the onset to instability:

$$
u_{t}+\Delta^{2} u+2 \Delta u+\alpha u+b|\nabla u|^{2}+u^{3}=0
$$

where $\alpha$ and $b$ are arbitrary constants. In the case of $b=0$, it is the usual Swift-Hohenberg equation. The additional term $b|\nabla u|^{2}$ arises in the study of various pattern formation phenomena involving some kind of phase turbulence or phase transition that breaks the symmetry $u \rightarrow-u$. For more references, one can see [6-8] and the references therein. The dynamical properties of the S-H equation are important for the studies of pattern formation and global attractors, and the stability of stationary solution and pattern selections of the S-H equation have been extensively investigated; see [9-11].

It turns out that a stochastic equation can conform to physical phenomena better in some cases. These random perturbations are intrinsic effects in a variety of settings and spatial scales. In fact, when the Rayleigh number is near thermal equilibrium, the influence of small noise or molecular noise is detected in various convection experiments [12, 13].

(c) The Author(s) 2017. This article is distributed under the terms of the Creative Commons Attribution 4.0 International License (http://creativecommons.org/licenses/by/4.0/), which permits unrestricted use, distribution, and reproduction in any medium, provided you give appropriate credit to the original author(s) and the source, provide a link to the Creative Commons license, and indicate if changes were made. 
As the effect of thermal fluctuations on the onset of convective motion into the Bénard system is considered, the stochastic local S-H equation with additive noise [1] is proposed:

$$
u_{t}=\mu u-\left(1+\partial_{x x}\right)^{2} u-u^{3}+\sigma \xi
$$

Furthermore, a local stochastic S-H equation driven by multiplicative noise arises when the effects of small possible noise from $\mu$ is considered [14]:

$$
u_{t}=\mu u-\left(1+\partial_{x x}\right)^{2} u-u^{3}+\sigma u \circ \xi
$$

where $\sigma>0$, and $\xi=\frac{d W}{d t}$ is the generalized derivative of a real-valued Brownian motion.

There have been a lot of outstanding work and important results related to the existence and uniqueness of solution and attractors for stochastic partial differential equations. For research progress on these aspects, we refer to [15-19]. Until now, there are few results on the dynamics behaviors of the stochastic local modified Swift-Hohenberg equation with multiplicative noise in Stratonovich sense. This is our main purpose. After making use of the O-U transform and changing the stochastic equation into the corresponding deterministic equation with random parameter, we obtain uniform a priori estimates under some additional assumptions and prove the existence of global random attractor for the random dynamical system associated with the stochastic local modified Swift-Hohenberg equation. It allows us to overcome the computational difficulties according to the properties of the local modified Swift-Hohenberg equation. In particular, showing the existence of a random attractor needs a lot of technical skills to obtain the desired results.

In this paper, we consider the following one-dimensional stochastic local modified SwiftHohenberg equation with multiplicative noise:

$$
d u+\left(\Delta^{2} u+2 \Delta u+\alpha u+b\left|u_{x}\right|^{2}-u^{3}\right) d t=\sigma u \circ d W(t)
$$

with initial condition

$$
u(x, 0)=u_{0}(x), \quad x \in D
$$

and the boundary conditions

$$
\left.u\right|_{\partial D}=\left.\Delta u\right|_{\partial D}=0, \quad x \in \partial D,
$$

where $D$ is a bounded open interval, $|b| \ll 1$ is a constant, $\Delta u$ means $u_{x x}$, and $\Delta^{2} u$ means $u_{x x x x}$.

An outline of this paper is as follows. We devote Section 2 to recall some definitions and results referred to global random attractors and to present some notation. In Section 3, we not only introduce the $\mathrm{O}-\mathrm{U}$ transformer, but also obtain uniform a priori estimates of the solution for the stochastic local modified Swift-Hohenberg equation. Finally, the proof of the main theorem on the existence of global random attractor is presented in Section 4.

\section{Preliminaries}

There are many research results on random attractors and related issues. For simplicity of the structure of the article, we only list the definitions; for the relevant theorems, we refer 
to [20, 21], and so on. Let $\left(X,\|\cdot\|_{X}\right)$ be a completely separable Hilbert space with Borel $\sigma$-algebra $\mathscr{B}(X)$, and let $\left(\Omega, \mathcal{F}, \mathbb{P},\left(\theta_{t}\right)_{t \in \mathbb{R}}\right)$ be an ergodic metric dynamical system.

Definition 2.1 (See $[15,16])$ A measurable mapping

$$
\varphi: \mathbb{R}^{+} \times \Omega \times X \rightarrow X, \quad(t, \omega, x) \mapsto \varphi(t, \omega, x),
$$

has the cocycle property:

(1) $\varphi(0, \omega, x)$ is the identity mapping on $X$;

(2) $\varphi(t+s, \omega, x)=\varphi\left(t, \theta_{s} \omega, \varphi(s, \omega, x)\right)$ for all $s, t \geq 0, x \in X$, and $\omega \in \Omega$.

We call $\varphi$ a random dynamical system (RDS) on $X$ over $\left(\Omega, \mathcal{F}, \mathbb{P},\left(\theta_{t}\right)_{t \in \mathbb{R}}\right)$. Furthermore, the $\operatorname{RDS} \varphi$ is continuous if $\varphi(t, \omega, \cdot)=\varphi(t, \omega): X \rightarrow X$ is continuous for all $t \geq 0$.

Definition 2.2 (See $[15,16]$ ) A random compact set $\mathcal{A}(\omega)$ is said to be a random attractor for RDS $\varphi$ if the following conditions hold:

(1) pullback attracting property:

$$
\lim _{t \rightarrow+\infty} d\left(\varphi\left(t, \theta_{-t} \omega, \mathscr{D}\left(\theta_{-t} \omega\right)\right) ; \mathcal{A}(\omega)\right)=0,
$$

(2) the invariance property: $\varphi(t, \omega, \mathcal{A}(\omega))=\mathcal{A}\left(\theta_{t} \omega\right)$ for $\mathbb{P}$-a.e. $\omega \in \Omega$ and all $t \geq 0$.

Remark Let $(\Omega, \mathcal{F}, \mathbb{P})$ be a probability space with Wiener measure $\mathbb{P}$. The Wiener shift $\left(\theta_{t}\right)_{t \in \mathbb{R}}$ is defined by

$$
\theta_{s} \omega(t)=\omega(t+s)-\omega(s), \quad t, s \in \mathbb{R}
$$

Then $\left(\Omega, \mathcal{F}, \mathbb{P},\left(\theta_{t}\right)_{t \in \mathbb{R}}\right)$ is an ergodic metric dynamical system; see [20].

For the convenience of the following contents, we introduce some functional spaces and some notations. $L^{q}(D)$ is the Lebesgue space with norm $\|\cdot\|_{L^{q}}$, and $\|\cdot\|_{L^{2}}=\|\cdot\|$. Particularly, $\|u\|_{L^{\infty}}=\operatorname{ess}_{\sup } \sec _{x \in D}|u(x)|$ for $q=\infty$.

$H^{\sigma}(D)$ is the Sobolev space $\left\{u \in L^{2}(D), D^{k} u \in L^{2}(D), k \leq \sigma\right\}$ with norm $\|\cdot\|_{H^{\sigma}}=\|\cdot\|_{\sigma}$. Especially, $H_{0}^{2}(D)$ is the Sobolev space $\left\{u \in L^{2}(D), D^{k} u \in L^{2}(D), k \leq 2,\left.\frac{\partial u}{\partial n}\right|_{x \in \partial D}=0\right\}$.

For notational simplicity, $C$ is a generic constant that may take various values from line to line; $\Delta v$ means $v_{x x}$, and $\Delta^{2} v$ means $v_{x x x x}$.

\section{Uniform a priori estimates of solution}

In this section, we mainly show uniform a priori estimates of a solution for the stochastic local modified Swift-Hohenberg equation.

The original equation (1.4) can be rewritten as follows:

$$
u(t)=u_{0}-\int_{0}^{t}\left(\alpha u+2 \Delta u+\Delta^{2} u+b\left|u_{x}\right|^{2}-u^{3}\right) d s+\sigma \int_{0}^{t} u(s) \circ d W(s) .
$$

We now introduce an O-U process $z\left(\theta_{t} \omega\right)$ that solves the following Itô equation:

$$
d z+z d t=\sigma d W(t)
$$


By $[20,22]$, the random variable $z\left(\theta_{t} \omega\right)$ is tempered, and for every $\omega \in \tilde{\Omega}, t \mapsto z\left(\theta_{t} \omega\right)$ is continuous with respect to $t$. Especially, the properties $\lim _{t \rightarrow \pm \infty} \frac{\left|z\left(\theta_{t} \omega\right)\right|}{|t|}=0$ and $\lim _{t \rightarrow \pm \infty} \frac{\int_{0}^{t} z\left(\theta_{s} \omega\right) d s}{t}=0$ hold.

Let $v=e^{-z\left(\theta_{t}(\omega)\right.} u(t)$. Using the Itô equation, combined with the original equation (1.4), we get

$$
\begin{aligned}
d v(t) & =e^{-z\left(\theta_{t}(\omega)\right.} d u(t)-u(t) e^{-z\left(\theta_{t}(\omega)\right.} \circ d\left(z\left(\theta_{t} \omega\right)\right) \\
& =-\left(\Delta^{2} v+2 \Delta v+\alpha v+e^{2 z\left(\theta_{t} \omega\right)} v^{3}+b e^{z\left(\theta_{t}(\omega)\right.} v_{x}^{2}\right) d t+z\left(\theta_{t} \omega\right) v(t) d t
\end{aligned}
$$

Thus, we consider the following system:

$$
\begin{aligned}
& \frac{d v}{d t}+\left(\alpha-z\left(\theta_{t} \omega\right)\right) v+2 \Delta v+\Delta^{2} v+e^{2 z\left(\theta_{t} \omega\right)} v^{3}+b e^{z\left(\theta_{t} \omega\right)} v_{x}^{2}=0, \\
& v(x, 0)=e^{-z(\omega)} u_{0}, \\
& \left.v\right|_{\partial D}=\left.\Delta v\right|_{\partial D}=0 .
\end{aligned}
$$

Similarly to $[8,23]$, by the Galerkin method and some a priori estimates we can prove that $v\left(t, \omega, v_{0}\right)$ is unique and continuous with respect to initial value $v_{0}$ in $H_{0}^{2}(D)$ for $\mathbb{P}$-a.e. $\omega \in \Omega$, where $v\left(t, \omega, v_{0}\right)$ is the solution of system (3.1)-(3.3). Define the continuous random dynamical system $\{\psi(t)\}_{t \geq 0}$ by

$$
\psi\left(t, \omega, v_{0}\right)=v\left(t, \omega, v_{0}\right)
$$

for all $v_{0} \in H_{0}^{2}(D), t \geq 0$, and $\omega \in \Omega$. Furthermore, setting $u\left(t, \omega, u_{0}\right)=e^{z\left(\theta_{t} \omega\right)} v\left(t, \omega, v_{0}\right)$, we have

$$
\phi\left(t, \omega, u_{0}\right)=u\left(t, \omega, u_{0}\right)=e^{z\left(\theta_{t} \omega\right)} v\left(t, \omega, v_{0}\right)=e^{z\left(\theta_{t} \omega\right)} \psi\left(t, \omega, v_{0}\right) .
$$

Then $\phi$ is a continuous random dynamical system on $H_{0}^{2}(D)$. It is straightforward to show that the existence of a random attractor for $\phi$ is equivalent to the existence of a random attractor for $\psi$.

In the following, provided that $\mathscr{D}$ is a collection of tempered random subsets of $H_{0}^{2}(D)$, we will prove the existence of an absorbing set in $H_{0}^{2}(D)$.

Lemma 3.1 Provided that $v_{0} \in B=\{B(\omega)\}_{\omega} \subset \mathscr{D}$, there exist a random radius $\rho_{1}(\omega)>0$ and $T_{1 B}(\omega)>0$ for $\mathbb{P}$-a.e. $\omega \in \Omega$ such that

$$
\left\|v\left(t, \theta_{-t} \omega, v_{0}\left(\theta_{-t} \omega\right)\right)\right\|^{2} \leq \rho_{1}(\omega), \quad t>T_{1 B}(\omega)
$$

Proof Taking the inner product of equation (3.1) with $v$, we have

$$
\begin{aligned}
& \frac{1}{2} \frac{d}{d t}\|v\|^{2}+\left(\alpha-z\left(\theta_{t} \omega\right)\right)\|v(t)\|^{2}+(2 \Delta v, v)+\|\Delta v\|^{2}+\left(e^{2 z\left(\theta_{t} \omega\right)} v^{3}, v\right) \\
& \quad+\left(b e^{z\left(\theta_{t} \omega\right)} v_{x}^{2}, v\right)=0 .
\end{aligned}
$$


Noticing that $e^{2 z\left(\theta_{t} \omega\right)}\left(v^{3}, v\right)=e^{2 z\left(\theta_{t} \omega\right)}\|v\|_{L^{4}}^{4}$ and applying the Hölder inequality and $\epsilon$-Young inequality, we get

$$
|(2 \Delta v, v)| \leq \frac{1}{4}\|\Delta v\|^{2}+4\|v\|^{2} .
$$

Now, we deal with the last term on the left side of equation (3.5). By integration by parts we have

$$
\int_{D} b e^{z\left(\theta_{t} \omega\right)} v_{x}^{2} v d x=-b e^{z\left(\theta_{t} \omega\right)} \int_{D} v\left(v_{x} v\right)_{x} d x=-b e^{z\left(\theta_{t} \omega\right)} \int_{D}\left(v^{2} v_{x x}+v v_{x}^{2}\right) d x,
$$

and thus

$$
b e^{z\left(\theta_{t} \omega\right)} \int_{D} v_{x}^{2} \nu d x=-\frac{b}{2} e^{z\left(\theta_{t} \omega\right)} \int_{D} v^{2} v_{x x} d x
$$

Applying the Hölder inequality and $\epsilon$-Young inequality again, we get

$$
\left|b e^{z\left(\theta_{t} \omega\right)} \int_{D} v_{x}^{2} v d x\right| \leq b C e^{z\left(\theta_{t} \omega\right)}\|v\|_{L^{4}}^{2}\left\|v_{x x}\right\| \leq \frac{1}{4}\left\|v_{x x}\right\|^{2}+b^{2} C e^{2 z\left(\theta_{t} \omega\right)}\|v\|_{L^{4}}^{4} .
$$

Taking $b$ small enough such that $b^{2} C \leq \frac{1}{2}$, we obtain

$$
\left|b e^{z\left(\theta_{t}(\omega)\right.} \int_{D} v_{x}^{2} v d x\right| \leq \frac{1}{4}\left\|v_{x x}\right\|^{2}+\frac{1}{2} e^{2 z\left(\theta_{t} \omega\right)}\|v\|_{L^{4}}^{4} .
$$

Putting all these inequalities together, we have

$$
\begin{aligned}
& \frac{1}{2} \frac{d}{d t}\|v\|^{2}+\frac{1}{2}\|\Delta v\|^{2}+\left(\beta-z\left(\theta_{t} \omega\right)\right)\|v\|^{2}+(\alpha-\beta-4)\|v\|^{2} \\
& \quad+\frac{1}{2} e^{2 z\left(\theta_{t} \omega\right)}\|v\|_{L^{4}}^{4} \leq 0
\end{aligned}
$$

where $\beta>0$ is a constant such that $\alpha-\beta-4<0$.

By the Sobolev imbedding $L^{4}(D) \subset L^{2}(D)$ we get

$$
\begin{aligned}
& \frac{d}{d t}\|v\|^{2}+2\left(\beta-z\left(\theta_{t} \omega\right)\right)\|v\|^{2}+\|\Delta v\|^{2} \\
& \quad \leq-e^{2 z\left(\theta_{t} \omega\right)}\|v\|_{L^{4}}^{4}-2 C(\alpha-\beta-4)\|v\|_{L^{4}}^{2} .
\end{aligned}
$$

We can change the right-hand side of this inequality as follows:

$$
\begin{aligned}
& -e^{2 z\left(\theta_{t} \omega\right)}\|v\|_{L^{4}}^{4}-2 C(\alpha-\beta-4)\|v\|_{L^{4}}^{2} \\
& \quad=-e^{2 z\left(\theta_{t} \omega\right)}\left(\|v\|_{L^{4}}^{2}+C e^{-2 z\left(\theta_{t} \omega\right)}(\alpha-\beta-4)\right)^{2}+C(\alpha-\beta-4)^{2} e^{-2 z\left(\theta_{t} \omega\right)} .
\end{aligned}
$$

Then we have

$$
\frac{d}{d t}\|v\|^{2}+2\left(\beta-z\left(\theta_{t} \omega\right)\right)\|v\|^{2}+\|\Delta v\|^{2} \leq C(\alpha-\beta-4)^{2} e^{-2 z\left(\theta_{t} \omega\right)} .
$$


By the Gronwall inequality we have

$$
\begin{aligned}
\left\|v\left(t, \omega, v_{0}(\omega)\right)\right\|^{2} \leq & e^{-2 \beta t+\int_{0}^{t} 2 z\left(\theta_{\tau} \omega\right) d \tau}\left\|v_{0}(\omega)\right\|^{2} \\
& +C(\alpha-\beta-4)^{2} \int_{0}^{t} e^{-2 z\left(\theta_{s} \omega\right)-2 \beta(t-s)+\int_{s}^{t} 2 z\left(\theta_{\tau} \omega\right) d \tau} d s .
\end{aligned}
$$

Furthermore, replacing $\omega$ with $\theta_{-t} \omega$ in (3.8), then we have

$$
\begin{aligned}
\| v(t, & \left.\theta_{-t} \omega, v_{0}\left(\theta_{-t} \omega\right)\right) \|^{2} \\
\leq & e^{-2 t\left(\beta-\frac{\int_{-t}^{0} 2 z\left(\theta_{\tau} \omega\right) d \tau}{t}\right)}\left\|v_{0}\left(\theta_{-t} \omega\right)\right\|^{2} \\
& +C(\alpha-\beta-4)^{2} \int_{0}^{t} e^{-2 z\left(\theta_{s-t} \omega\right)-2 \beta(t-s)+\int_{s-t}^{0} 2 z\left(\theta_{\tau} \omega\right) d \tau} d s \\
= & e^{-2 t\left(\beta-\frac{\int_{-t}^{0} 2 z\left(\theta_{\tau} \omega\right) d \tau}{t}\right)}\left\|v_{0}\left(\theta_{-t} \omega\right)\right\|^{2}+C(\alpha-\beta-4)^{2} \int_{-t}^{0} e^{-2 z\left(\theta_{s} \omega\right)+2 \beta s+\int_{s}^{0} 2 z\left(\theta_{\tau} \omega\right) d \tau} d s \\
\leq & e^{-2 t\left(\beta-\frac{\int_{-t}^{0} 2 z\left(\theta_{\tau} \omega\right) d \tau}{t}\right)}\left\|v_{0}\left(\theta_{-t} \omega\right)\right\|^{2} \\
& +C(\alpha-\beta-4)^{2} \int_{-\infty}^{0} e^{2 s\left(\beta-\frac{z\left(\theta_{s} \omega\right)}{s}+\frac{\int_{s}^{0} z\left(\theta_{\tau} \omega\right) d \tau}{s}\right)} d s .
\end{aligned}
$$

Because of the properties of $z\left(\theta_{t} \omega\right)$, there exists $T_{1 B}(\omega)>0$ such that, for all $t \geq T_{1 B}(\omega)$,

$$
\frac{\int_{-t}^{0} 2 z\left(\theta_{\tau} \omega\right) d \tau}{t} \leq \frac{\beta}{2}
$$

It follows that

$$
e^{-2 t\left(\beta-\frac{\int_{-t}^{0} 2 z\left(\theta_{\tau} \omega\right) d \tau}{t}\right)}\left\|v_{0}\left(\theta_{-t} \omega\right)\right\|^{2} \leq e^{-\beta t}\left\|v_{0}\left(\theta_{-t} \omega\right)\right\|^{2} .
$$

The random set $\mathscr{D}$ is tempered, which implies the boundedness of the first term on the right-hand side of (3.9). The second term on the right-hand side of (3.9) is convergent.

Thus, there exist $T_{1 B}(\omega)>0$ and a random variable $\rho_{1}(\omega)$ such that, for $\mathbb{P}$-a.e. $\omega \in \Omega$ and all $t>T_{1 B}(\omega)$,

$$
\left\|v\left(t, \theta_{-t} \omega, v_{0}\left(\theta_{-t} \omega\right)\right)\right\|^{2} \leq \rho_{1}(\omega)
$$

Lemma 3.2 Provided that $v_{0} \in B=\{B(\omega)\}_{\omega} \subset \mathscr{D}$, there exist a random radius $\rho_{2}(\omega)>0$ and $T_{2 B}(\omega)>0$ for $\mathbb{P}$-a.e. $\omega \in \Omega$ such that

$$
\int_{t}^{t+1}\left\|\Delta v\left(s, \theta_{-t-1} \omega, v_{0}\left(\theta_{-t-1} \omega\right)\right)\right\|^{2} d s \leq \rho_{2}(\omega), \quad t>T_{2 B}(\omega)
$$

Proof Multiplying equation (3.7) by $e^{2 \beta t-\int_{0}^{t} 2 z\left(\theta_{\tau} \omega\right) d \tau}$, we get

$$
\begin{gathered}
\frac{d}{d t}\left[e^{2 \beta t-\int_{0}^{t} 2 z\left(\theta_{\tau} \omega\right) d \tau}\|v\|^{2}\right]+e^{2 \beta t-\int_{0}^{t} 2 z\left(\theta_{\tau} \omega\right) d \tau}\|\Delta v\|^{2} \\
\quad \leq C(\alpha-\beta-4)^{2} e^{-2 z\left(\theta_{t} \omega\right)+2 \beta t-\int_{0}^{t} 2 z\left(\theta_{\tau} \omega\right) d \tau} .
\end{gathered}
$$


Set $\widehat{T} \leq t \leq t+1$. Integrating from $\widehat{T}$ to $t$, we have

$$
\begin{aligned}
& e^{2 \beta t-\int_{0}^{t} 2 z\left(\theta_{\tau} \omega\right) d \tau}\|v(t)\|^{2}+\int_{\widehat{T}}^{t} e^{2 \beta s-\int_{0}^{s} 2 z\left(\theta_{\tau} \omega\right) d \tau}\|\Delta v(s)\|^{2} d s \\
& \quad \leq C(\alpha-\beta-4)^{2} \int_{\widehat{T}}^{t} e^{-2 z\left(\theta_{s} \omega\right)+2 \beta s-\int_{0}^{s} 2 z\left(\theta_{\tau} \omega\right) d \tau} d s+e^{2 \beta \widehat{T}-\int_{0}^{\widehat{T}} 2 z\left(\theta_{\tau} \omega\right) d \tau}\left\|v\left(\widehat{T}, \omega, v_{0}(\omega)\right)\right\|^{2} .
\end{aligned}
$$

Multiplying this inequality by $e^{-2 \beta t+\int_{0}^{t} 2 z\left(\theta_{\tau} \omega\right) d \tau}$ and getting rid of the first term, we obtain

$$
\begin{aligned}
\int_{\widehat{T}}^{t} e^{2 \beta(s-t)+\int_{s}^{t} 2 z\left(\theta_{\tau} \omega\right) d \tau}\|\Delta v(s)\|^{2} d s \\
\quad \leq e^{2 \beta(\widehat{T}-t)+\int_{\widehat{T}}^{t} 2 z\left(\theta_{\tau} \omega\right) d \tau}\left\|v\left(\widehat{T}, \omega, v_{0}(\omega)\right)\right\|^{2} \\
\quad+C(\alpha-\beta-4)^{2} \int_{\widehat{T}}^{t} e^{-2 z\left(\theta_{s} \omega\right)+2 \beta(s-t)+\int_{s}^{t} 2 z\left(\theta_{\tau} \omega\right) d \tau} d s .
\end{aligned}
$$

Now, substituting $t$ for $\widehat{T}$ in (3.8), we obtain

$$
\begin{aligned}
\left\|v\left(\widehat{T}, \omega, v_{0}(\omega)\right)\right\|^{2} \leq & e^{-2 \beta \widehat{T}+\int_{0}^{\widehat{T}} 2 z\left(\theta_{\tau} \omega\right) d \tau}\left\|v_{0}(\omega)\right\|^{2} \\
& +C(\alpha-\beta-4)^{2} \int_{0}^{\widehat{T}} e^{-2 z\left(\theta_{s} \omega\right)-2 \beta(\widehat{T}-s)+\int_{s}^{\widehat{T}} 2 z\left(\theta_{\tau} \omega\right) d \tau} d s .
\end{aligned}
$$

If we plug (3.13) back into (3.12), we have

$$
\begin{aligned}
& \int_{\widehat{T}}^{t} e^{2 \beta(s-t)+\int_{s}^{t} 2 z\left(\theta_{\tau} \omega\right) d \tau}\left\|\Delta v\left(s, \omega, v_{0}(\omega)\right)\right\|^{2} d s \\
& \quad \leq e^{-2 \beta t+\int_{0}^{t} 2 z\left(\theta_{\tau} \omega\right) d \tau}\left\|v_{0}(\omega)\right\|^{2} \\
& \quad+C(\alpha-\beta-4)^{2} \int_{0}^{t} e^{-2 z\left(\theta_{s} \omega\right)-2 \beta(t-s)+\int_{s}^{t} 2 z\left(\theta_{\tau} \omega\right) d \tau} d s .
\end{aligned}
$$

Replacing $\omega$ with $\theta_{-t} \omega$ in (3.14), we get

$$
\begin{gathered}
\int_{\widehat{T}}^{t} e^{2 \beta(s-t)+\int_{s}^{t} 2 z\left(\theta_{\tau-t} \omega\right) d \tau}\left\|\Delta v\left(s, \theta_{-t} \omega, v_{0}\left(\theta_{-t} \omega\right)\right)\right\|^{2} d s \\
\leq C(\alpha-\beta-4)^{2} \int_{0}^{t} e^{-2 z\left(\theta_{s-t} \omega\right)-2 \beta(t-s)+\int_{s}^{t} 2 z\left(\theta_{\tau-t} \omega\right) d \tau} d s \\
\quad+e^{-2 \beta t+\int_{0}^{t} 2 z\left(\theta_{\tau-t} \omega\right) d \tau}\left\|\nu_{0}\left(\theta_{-t} \omega\right)\right\|^{2} .
\end{gathered}
$$

In order to obtain the result, we need to substitute $\widehat{T}$ for $t$ and $t$ for $t+1$ in (3.15) as follows:

$$
\begin{aligned}
& \int_{t}^{t+1} e^{2 \beta(s-t-1)+\int_{s}^{t+1} 2 z\left(\theta_{\tau-t-1} \omega\right) d \tau}\left\|\Delta v\left(s, \theta_{-t-1} \omega, v_{0}\left(\theta_{-t-1} \omega\right)\right)\right\|^{2} d s \\
& \quad \leq e^{-2 \beta(t+1)+\int_{0}^{t+1} 2 z\left(\theta_{\tau-t-1} \omega\right) d \tau}\left\|v_{0}\left(\theta_{-t-1} \omega\right)\right\|^{2} \\
& \quad+C(\alpha-\beta-4)^{2} \int_{0}^{t+1} e^{-2 z\left(\theta_{s-t-1} \omega\right)-2 \beta(t+1-s)+\int_{s}^{t+1} 2 z\left(\theta_{\tau-t-1} \omega\right) d \tau} d s
\end{aligned}
$$


that is,

$$
\begin{aligned}
& \int_{t}^{t+1} e^{2 \beta(s-t-1)+\int_{s-t-1}^{0} 2 z\left(\theta_{\tau} \omega\right) d \tau}\left\|\Delta v\left(s, \theta_{-t-1} \omega, v_{0}\left(\theta_{-t-1} \omega\right)\right)\right\|^{2} d s \\
& \leq e^{-2(t+1)\left(\beta-\frac{\int_{-t-1}^{0} 2 z\left(\theta_{\tau} \omega\right) d \tau}{2(t+1)}\right)}\left\|v_{0}\left(\theta_{-t-1} \omega\right)\right\|^{2} \\
& \quad+C(\alpha-\beta-4)^{2} \int_{-t-1}^{0} e^{-2 z\left(\theta_{s} \omega\right)+2 \beta s+\int_{s}^{0} 2 z\left(\theta_{\tau} \omega\right) d \tau} d s \\
& \leq e^{-2(t+1)\left(\beta-\frac{\int_{-t-1}^{0} 2 z\left(\theta_{\tau} \omega\right) d \tau}{2(t+1)}\right)}\left\|v_{0}\left(\theta_{-t-1} \omega\right)\right\|^{2} \\
& \quad+C(\alpha-\beta-4)^{2} \int_{-\infty}^{0} e^{-2 z\left(\theta_{s} \omega\right)+2 \beta s+\int_{s}^{0} 2 z\left(\theta_{\tau} \omega\right) d \tau} d s .
\end{aligned}
$$

According to the properties of $z\left(\theta_{t} \omega\right)$, when $-1 \leq s-t-1 \leq 0$, we can deduce

$$
\begin{aligned}
& \int_{t}^{t+1} e^{2 \beta(s-t-1)+\int_{s-t-1}^{0} 2 z\left(\theta_{\tau} \omega\right) d \tau}\left\|\Delta v\left(s, \theta_{-t-1} \omega, v_{0}\left(\theta_{-t-1} \omega\right)\right)\right\|^{2} d s \\
& \quad \geq e^{-2 \beta-2 \max _{-1 \leq \tau \leq 0}\left|z\left(\theta_{\tau} \omega\right)\right|} \int_{t}^{t+1}\left\|\Delta v\left(s, \theta_{-t-1} \omega, v_{0}\left(\theta_{-t-1} \omega\right)\right)\right\|^{2} d s .
\end{aligned}
$$

Combining estimates (3.16) and (3.17), we prove that there exist a random variable $\rho_{2}(\omega)$ and $T_{2 B}(\omega)>0$ such that, for $\mathbb{P}$-a.e. $\omega \in \Omega$ and all $t>T_{2 B}(\omega)$,

$$
\int_{t}^{t+1}\left\|\Delta v\left(s, \theta_{-t-1} \omega, v_{0}\left(\theta_{-t-1} \omega\right)\right)\right\|^{2} d s \leq \rho_{2}(\omega)
$$

The proof of the lemma is now complete.

\section{The existence of random attractor}

Lemma 4.1 Provided that $v_{0} \in B=\{B(\omega)\}_{\omega} \subset \mathscr{D}$, there exist a random radius $\rho_{3}(\omega)>0$ and $T_{3 B}(\omega)>0$ for $\mathbb{P}$-a.e. $\omega \in \Omega$ such that

$$
\left\|\Delta v\left(t, \theta_{-t} \omega, v_{0}\left(\theta_{-t} \omega\right)\right)\right\|^{2} \leq \rho_{3}(\omega), \quad t>T_{3 B}(\omega) .
$$

Proof Taking the inner product of equation (3.1) with $\Delta^{2} v$, we have

$$
\begin{aligned}
& \frac{1}{2} \frac{d}{d t}\|\Delta v\|^{2}+\left\|\Delta^{2} v\right\|^{2}+\left(2 \Delta v, \Delta^{2} v\right)+\left(\alpha-z\left(\theta_{t} \omega\right)\right)\|\Delta v\|^{2} \\
& +\left(e^{2 z\left(\theta_{t}(\omega)\right.} v^{3}, \Delta^{2} v\right)+\left(b e^{z\left(\theta_{t} \omega\right)}\left(v_{x}\right)^{2}, \Delta^{2} v\right)=0 .
\end{aligned}
$$

Applying the Hölder inequality and $\epsilon$-Young inequality, we get

$$
\left|\left(2 \Delta v, \Delta^{2} v\right)\right| \leq \frac{1}{4}\left\|\Delta^{2} v\right\|^{2}+4\|\Delta v\|^{2} .
$$


By the Gagliardo-Nirenberg inequality $\|v\|_{L^{6}} \leq C\|v\|^{\frac{11}{12}}\left\|\Delta^{2} v\right\|^{\frac{1}{12}}$ (see [8]) and the $\epsilon$-Young inequality we have

$$
\begin{aligned}
\left|\left(e^{2 z\left(\theta_{t} \omega\right)} v^{3}, \Delta^{2} v\right)\right| & \leq C e^{2 z\left(\theta_{t} \omega\right)}\|v\|_{L^{6}}^{3}\left\|\Delta^{2} v\right\| \leq C e^{2 z\left(\theta_{t} \omega\right)}\left\|\Delta^{2} v\right\| \cdot\|v\|^{\frac{11}{4}}\left\|\Delta^{2} v\right\|^{\frac{1}{4}} \\
& \leq \eta_{1}\left\|\Delta^{2} v\right\|^{2}+C\left(\eta_{1}\right) e^{\frac{16 z\left(\theta_{t} \omega\right)}{3}}\|v\|^{\frac{22}{3}} .
\end{aligned}
$$

Similarly, by the Gagliardo-Nirenberg inequality $\left\|v_{x}\right\|_{L^{4}} \leq C\|v\| \frac{11}{16}\left\|\Delta^{2} v\right\|^{\frac{5}{16}}$ and the $\epsilon$ Young inequality again, we obtain

$$
\begin{aligned}
\left|\left(b e^{z\left(\theta_{t}(\omega)\right.} v_{x}^{2}, \Delta^{2} v\right)\right| & \leq|b| C e^{z\left(\theta_{t} \omega\right)}\left\|\Delta^{2} v\right\|\left\|v_{x}\right\|_{L^{4}}^{2} \leq|b| C e^{z\left(\theta_{t} \omega\right)}\left\|\Delta^{2} v\right\|^{\frac{13}{8}}\|v\|^{\frac{11}{8}} \\
& \leq \eta_{2}\left\|\Delta^{2} v\right\|^{2}+C\left(\eta_{2}\right) e^{\frac{16}{3} z\left(\theta_{t} \omega\right)}\|v\|^{\frac{22}{3}} .
\end{aligned}
$$

Taking $\eta_{1}=\eta_{2}=\frac{1}{4}$, we deduce

$$
\begin{gathered}
\frac{d}{d t}\|\Delta v\|^{2}+2\left(\beta-z\left(\theta_{t} \omega\right)\right)\|\Delta v\|^{2}+\frac{1}{2}\left\|\Delta^{2} v\right\|^{2} \\
\quad \leq C e^{\frac{16}{3} z\left(\theta_{t} \omega\right)}\|v\|^{\frac{22}{3}}+2(4-\alpha+\beta)\|\Delta v\|^{2} .
\end{gathered}
$$

Integrating (4.3) from $s$ to $t+1$ with respect to $t$, we have

$$
\begin{aligned}
& \left\|\Delta v\left(t+1, \omega, v_{0}(\omega)\right)\right\|^{2} \\
& \leq\left\|\Delta v\left(s, \omega, v_{0}(\omega)\right)\right\|^{2}+2 \int_{s}^{t+1}\left(z\left(\theta_{\tau} \omega\right)-\beta\right)\left\|\Delta v\left(\tau, \omega, v_{0}(\omega)\right)\right\|^{2} d \tau \\
& \quad+\int_{s}^{t+1}\left[C e^{\frac{16}{3} z\left(\theta_{\tau} \omega\right)}\left\|v\left(\tau, \omega, v_{0}(\omega)\right)\right\|^{\frac{22}{3}}+2(4-\alpha+\beta)\left\|\Delta v\left(\tau, \omega, v_{0}(\omega)\right)\right\|^{2}\right] d \tau .
\end{aligned}
$$

Integrating from $t$ to $t+1$ with respect to $s$ again, we get

$$
\begin{aligned}
\| \Delta & v\left(t+1, \omega, v_{0}(\omega)\right) \|^{2} \\
\leq & \int_{t}^{t+1}\left\|\Delta v\left(s, \omega, v_{0}(\omega)\right)\right\|^{2} d s+2 \int_{t}^{t+1}\left|\left(z\left(\theta_{\tau} \omega\right)-\beta\right)\right|\left\|\Delta v\left(\tau, \omega, v_{0}(\omega)\right)\right\|^{2} d \tau \\
& \quad+\int_{t}^{t+1} C e^{\frac{16}{3} z\left(\theta_{\tau} \omega\right)}\left\|v\left(\tau, \omega, v_{0}(\omega)\right)\right\|^{\frac{22}{3}} d \tau+2(4-\alpha+\beta) \int_{t}^{t+1}\left\|\Delta v\left(\tau, \omega, v_{0}(\omega)\right)\right\|^{2} d \tau \\
\leq & (9-2 \alpha+4 \beta) \int_{t}^{t+1}\left\|\Delta v\left(s, \omega, v_{0}(\omega)\right)\right\|^{2} d s+2 \int_{t}^{t+1}\left|z\left(\theta_{\tau} \omega\right)\right|\left\|\Delta v\left(\tau, \omega, v_{0}(\omega)\right)\right\|^{2} d \tau \\
\quad & +\int_{t}^{t+1} C e^{\frac{16}{3} z\left(\theta_{\tau} \omega\right)}\left\|v\left(\tau, \omega, v_{0}(\omega)\right)\right\|^{\frac{22}{3}} d \tau .
\end{aligned}
$$

Replacing $\omega$ with $\theta_{-t-1} \omega$ in (4.4), we have

$$
\begin{aligned}
& \left\|\Delta v\left(t+1, \theta_{-t-1} \omega, v_{0}\left(\theta_{-t-1} \omega\right)\right)\right\|^{2} \\
& \quad \leq(9-2 \alpha+4 \beta) \int_{t}^{t+1}\left\|\Delta v\left(s, \theta_{-t-1} \omega, v_{0}\left(\theta_{-t-1} \omega\right)\right)\right\|^{2} d s
\end{aligned}
$$




$$
\begin{aligned}
& +2 \int_{t}^{t+1}\left|z\left(\theta_{\tau-t-1} \omega\right)\right|\left\|\Delta v\left(\tau, \theta_{-t-1} \omega, v_{0}\left(\theta_{-t-1} \omega\right)\right)\right\|^{2} d \tau \\
& +\int_{t}^{t+1} C e^{\frac{16}{3} z\left(\theta_{\tau-t-1} \omega\right)}\left\|v\left(\tau, \theta_{-t-1} \omega, v_{0}\left(\theta_{-t-1} \omega\right)\right)\right\|^{\frac{22}{3}} d \tau
\end{aligned}
$$

According to Lemma 3.2, the first term on the right-hand side of the above inequality is bounded:

$$
(9-2 \alpha+4 \beta) \int_{t}^{t+1}\left\|\Delta v\left(s, \theta_{-t-1} \omega, v_{0}\left(\theta_{-t-1} \omega\right)\right)\right\|^{2} d s \leq|9-2 \alpha+4 \beta| \rho_{2}(\omega) .
$$

For the second term, taking into account the properties of $z\left(\theta_{t} \omega\right)$, we have $\left|z\left(\theta_{t} \omega\right)\right| \leq$ $e^{|t|} r(\omega)$. Then

$$
\begin{aligned}
& 2 \int_{t}^{t+1}\left|z\left(\theta_{\tau-t-1} \omega\right)\right|\left\|\Delta v\left(\tau, \theta_{-t-1} \omega, v_{0}\left(\theta_{-t-1} \omega\right)\right)\right\|^{2} d \tau \\
& \quad \leq 2 \int_{t}^{t+1} e^{|\tau-t-1|} r(\omega)\left\|\Delta v\left(\tau, \theta_{-t-1} \omega, v_{0}\left(\theta_{-t-1} \omega\right)\right)\right\|^{2} d \tau
\end{aligned}
$$

Noticing that $|\tau-t-1|<1$ and applying Lemma 3.2, we obtain

$$
2 \int_{t}^{t+1}\left|z\left(\theta_{\tau-t-1} \omega\right)\right|\left\|\Delta v\left(\tau, \theta_{-t-1} \omega, v_{0}\left(\theta_{-t-1} \omega\right)\right)\right\|^{2} d \tau \leq 2 \operatorname{er}(\omega) \rho_{2}(\omega) .
$$

Now, we estimate the last term. Replacing $t$ with $\tau$ in (3.8), we get

$$
\begin{aligned}
\left\|v\left(\tau, \omega, v_{0}(\omega)\right)\right\|^{2} \leq & e^{-2 \beta \tau+\int_{0}^{\tau} 2 z\left(\theta_{r} \omega\right) d r}\left\|v_{0}(\omega)\right\|^{2} \\
& +C(\alpha-\beta-4)^{2} \int_{0}^{\tau} e^{-2 z\left(\theta_{s} \omega\right)-2 \beta(\tau-s)+\int_{s}^{\tau} 2 z\left(\theta_{r} \omega\right) d r} d s .
\end{aligned}
$$

Substituting $\omega$ for $\theta_{-t-1} \omega$ in (4.6), we obtain

$$
\begin{aligned}
& \left\|v\left(\tau, \theta_{-t-1} \omega, v_{0}\left(\theta_{-t-1} \omega\right)\right)\right\|^{2} \\
& \quad \leq e^{-2 \beta \tau+\int_{0}^{\tau} 2 z\left(\theta_{r-t-1} \omega\right) d r}\left\|v_{0}\left(\theta_{-t-1} \omega\right)\right\|^{2} \\
& \quad+C(\alpha-\beta-4)^{2} \int_{0}^{\tau} e^{-2 z\left(\theta_{s-t-1} \omega\right)-2 \beta(\tau-s)+\int_{s}^{\tau} 2 z\left(\theta_{r-t-1} \omega\right) d r} d s .
\end{aligned}
$$

If we plug (4.7) back into (4.5), we obtain

$$
\begin{aligned}
& \int_{t}^{t+1} C e^{\frac{16}{3} z\left(\theta_{\tau-t-1} \omega\right)}\left[e^{-2 \beta \tau+\int_{0}^{\tau} 2 z\left(\theta_{r-t-1} \omega\right) d r}\left\|v_{0}\left(\theta_{-t-1} \omega\right)\right\|^{2}\right. \\
& \left.\quad+C(\alpha-\beta-4)^{2} \int_{0}^{\tau} e^{-2 z\left(\theta_{s-t-1} \omega\right)-2 \beta(\tau-s)+\int_{s}^{\tau} 2 z\left(\theta_{r-t-1} \omega\right) d r} d s\right]^{\frac{11}{3}} d \tau \\
& \leq \int_{t}^{t+1} C e^{\frac{16}{3} z\left(\theta_{\tau-t-1} \omega\right)}\left[\left(e^{-2 \beta \tau+\int_{0}^{\tau} 2 z\left(\theta_{r-t-1} \omega\right) d r}\left\|v_{0}\left(\theta_{-t-1} \omega\right)\right\|^{2}\right)^{\frac{11}{3}}\right. \\
& \left.\quad+C(\alpha-\beta-4)^{\frac{22}{3}}\left(\int_{0}^{\tau} e^{-2 z\left(\theta_{s-t-1} \omega\right)-2 \beta(\tau-s)+\int_{s}^{\tau} 2 z\left(\theta_{r-t-1} \omega\right) d r} d s\right)^{\frac{11}{3}}\right] d \tau
\end{aligned}
$$




$$
\begin{aligned}
= & \int_{t}^{t+1} C e^{\frac{16}{3} z\left(\theta_{\tau-t-1} \omega\right)}\left(e^{-2 \beta \tau+\int_{0}^{\tau} 2 z\left(\theta_{r-t-1} \omega\right) d r}\left\|v_{0}\left(\theta_{-t-1} \omega\right)\right\|^{2}\right)^{\frac{11}{3}} d \tau \\
& +C(\alpha-\beta-4)^{\frac{22}{3}} \\
& \times \int_{t}^{t+1} e^{\frac{16}{3} z\left(\theta_{\tau-t-1} \omega\right)}\left(\int_{0}^{\tau} e^{-2 z\left(\theta_{s-t-1} \omega\right)-2 \beta(\tau-s)+\int_{s}^{\tau} 2 z\left(\theta_{r-t-1} \omega\right) d r} d s\right)^{\frac{11}{3}} d \tau .
\end{aligned}
$$

Next, we estimate each term on the right-hand side of the last inequality. For the first term, we have

$$
\begin{aligned}
C & \int_{t}^{t+1}\left(e^{-2 \beta \tau+\int_{0}^{\tau} 2 z\left(\theta_{r-t-1} \omega\right) d r}\left\|v_{0}\left(\theta_{-t-1} \omega\right)\right\|^{2}\right)^{\frac{11}{3}} e^{\frac{16}{3} z\left(\theta_{\tau-t-1} \omega\right)} d \tau \\
& =C \int_{t}^{t+1}\left(e^{-2 \beta \tau+\int_{-t-1}^{\tau-t-1} 2 z\left(\theta_{r} \omega\right) d r}\left\|v_{0}\left(\theta_{-t-1} \omega\right)\right\|^{2}\right)^{\frac{11}{3}} e^{\frac{16}{3} z\left(\theta_{\tau-t-1} \omega\right)} d \tau \\
& =C \int_{-1}^{0}\left(e^{-2 \beta(\tau+1+t)+\int_{-t-1}^{\tau} 2 z\left(\theta_{r} \omega\right) d r}\left\|v_{0}\left(\theta_{-t-1} \omega\right)\right\|^{2}\right)^{\frac{11}{3}} e^{\frac{16}{3} z\left(\theta_{\tau} \omega\right)} d \tau \\
& \leq C e^{\frac{16}{3} \max _{-1 \leq \tau \leq 0}\left|z\left(\theta_{\tau} \omega\right)\right|} \int_{-1}^{0}\left(e^{-2 \beta(\tau+t+1)+\int_{-t-1}^{0} 2\left|z\left(\theta_{r} \omega\right)\right| d r}\right)^{\frac{11}{3}} d \tau \\
& =C e^{\frac{16}{3} \max _{-1 \leq \tau \leq 0}\left|z\left(\theta_{\tau} \omega\right)\right|} \int_{-1}^{0}\left(e^{-2 \beta(t+1)+\int_{-t-1}^{0} 2\left|z\left(\theta_{r} \omega\right)\right| d r}\right)^{\frac{11}{3}} \cdot e^{-\frac{22}{3} \beta \tau} d \tau \\
& =C e^{\frac{16}{3} \max _{-1 \leq \tau \leq 0}\left|z\left(\theta_{\tau} \omega\right)\right|}\left(e^{-2 \beta(t+1)+\int_{-t-1}^{0} 2\left|z\left(\theta_{r} \omega\right)\right| d r}\right)^{\frac{11}{3}} \cdot \int_{-1}^{0} e^{-\frac{22}{3} \beta \tau} d \tau \\
& \leq C e^{\frac{16}{3} \max _{-1 \leq \tau \leq 0}\left|z\left(\theta_{\tau} \omega\right)\right|}\left(e^{-2 \beta(t+1)+\int_{-t-1}^{0} 2\left|z\left(\theta_{r} \omega\right)\right| d r}\right)^{\frac{11}{3}},
\end{aligned}
$$

where the first inequality is due to the properties of $z\left(\theta_{t} \omega\right)$.

For the second term, similarly to the above method, as $t<\tau<t+1$, we have

$$
\begin{aligned}
& C(\alpha-\beta-4)^{\frac{22}{3}} \int_{t}^{t+1} e^{\frac{16}{3} z\left(\theta_{\tau-t-1} \omega\right)}\left(\int_{0}^{\tau} e^{-2 z\left(\theta_{s-t-1} \omega\right)-2 \beta(\tau-s)+\int_{s}^{\tau} 2 z\left(\theta_{r-t-1} \omega\right) d r} d s\right)^{\frac{11}{3}} d \tau \\
& \leq C(\alpha-\beta-4)^{\frac{22}{3}} \int_{t}^{t+1} e^{\frac{16}{3} z\left(\theta_{\tau-t-1} \omega\right)}\left(\int_{0}^{\tau} e^{-2 z\left(\theta_{s-t-1} \omega\right)+\int_{s-t-1}^{0} 2\left|z\left(\theta_{r} \omega\right)\right| d r-2 \beta(\tau-s)} d s\right)^{\frac{11}{3}} d \tau \\
& \leq C(\alpha-\beta-4)^{\frac{22}{3}} \int_{t}^{t+1} e^{\frac{16}{3} z\left(\theta_{\tau-t-1} \omega\right)}\left(\int_{-t-1}^{\tau-t-1} e^{-2 z\left(\theta_{s} \omega\right)+\int_{s}^{0} 2\left|z\left(\theta_{r} \omega\right)\right| d r-2 \beta(\tau-t-1-s)} d s\right)^{\frac{11}{3}} d \tau \\
& \leq C(\alpha-\beta-4)^{\frac{22}{3}} \\
& \quad \times \int_{t}^{t+1} e^{\frac{16}{3} z\left(\theta_{\tau-t-1} \omega\right)}\left(\int_{-t-1}^{0} e^{-2 z\left(\theta_{s} \omega\right)+2 \beta s+\int_{s}^{0} 2\left|z\left(\theta_{r} \omega\right)\right| d r+2 \beta} d s\right)^{\frac{11}{3}} d \tau
\end{aligned}
$$

where by the condition $t<\tau<t+1,-2 \beta(\tau-t)<0$, we get rid of the term $e^{-2 \beta(\tau-t)}$. Then the last inequality can be estimated as follows:

$$
\begin{gathered}
C(\alpha-\beta-4)^{\frac{22}{3}} \int_{t}^{t+1} e^{\frac{16}{3} z\left(\theta_{\tau-t-1} \omega\right)}\left(\int_{0}^{\tau} e^{-2 z\left(\theta_{s-t-1} \omega\right)-2 \beta(\tau-s)+\int_{s}^{\tau} 2 z\left(\theta_{r-t-1} \omega\right) d r} d s\right)^{\frac{11}{3}} d \tau \\
\leq C(\alpha-\beta-4)^{\frac{22}{3}} e^{\frac{22}{3} \beta} \int_{t}^{t+1} e^{\frac{16}{3} z\left(\theta_{\tau-t-1} \omega\right)}\left(\int_{-\infty}^{0} e^{-2 z\left(\theta_{s} \omega\right)+2 \beta s+\int_{s}^{0} 2\left|z\left(\theta_{r} \omega\right)\right| d r} d s\right)^{\frac{11}{3}} d \tau
\end{gathered}
$$




$$
\begin{aligned}
& \leq C(\alpha-\beta-4)^{\frac{22}{3}} e^{\frac{22}{3} \beta} \int_{-1}^{0} e^{\frac{16}{3} z\left(\theta_{\tau} \omega\right)}\left(\int_{-\infty}^{0} e^{-2 z\left(\theta_{s} \omega\right)+2 \beta s+\int_{s}^{0} 2\left|z\left(\theta_{r} \omega\right)\right| d r} d s\right)^{\frac{11}{3}} d \tau \\
& \leq C(\alpha-\beta-4)^{\frac{22}{3}} e^{\frac{22}{3} \beta} e^{\frac{16}{3} \max _{-1 \leq \tau \leq 0}\left|z\left(\theta_{\tau} \omega\right)\right|}\left(\int_{-\infty}^{0} e^{-2 z\left(\theta_{s} \omega\right)+2 \beta s+\int_{s}^{0} 2\left|z\left(\theta_{r} \omega\right)\right| d r} d s\right)^{\frac{11}{3}} .
\end{aligned}
$$

In summary, from estimates of (4.9) and (4.10) we obtain that the terms on the right-hand of inequality (4.8) are bounded. Therefore, we prove that there exist a random variable $\rho_{3}(\omega)$ and $T_{3 B}(\omega)>0$ such that for $\mathbb{P}$-a.e. $\omega \in \Omega$ and all $t>T_{3 B}(\omega)$,

$$
\left\|\Delta v\left(t+1, \theta_{-t-1} \omega, v_{0}\left(\theta_{-t-1} \omega\right)\right)\right\|^{2} \leq \rho_{3}(\omega) .
$$

This completes the proof.

Based on the above arguments, it is easy to deduce that there exists a random absorbing set for the random dynamical system generated by system (3.1)-(3.3) in $H_{0}^{2}(D)$.

Theorem 4.1 Assume that $v_{0} \in B=\{B(\omega)\}_{\omega} \subset \mathscr{D}$. There exists a random absorbing set $B^{*}(\omega)$ for the random dynamical system associated with system (3.1)-(3.3) in $H_{0}^{2}(D)$.

Proof We can take $T=\max \left\{T_{1 B}, T_{2 B}, T_{3 B}\right\}$ and $\rho(\omega)=\max \left\{\rho_{1}(\omega), \rho_{2}(\omega), \rho_{3}(\omega)\right\}$. Then, for all $t \geq T$ and $\mathbb{P}$-a.e. $\omega \in \Omega$, there exists a random absorbing set $B^{*}(\omega)$ for the random dynamical system associated with system (3.1)-(3.3) in $H_{0}^{2}(D)$.

Based on the above results, by Lemma 2.8 in [10] we claim that $\psi$ is asymptotically compact. Therefore, the existence of a random attractor for $\psi$ follows immediately from Theorem 2.2 in [15].

Theorem 4.2 Provided that $v_{0} \in B=\{B(\omega)\}_{\omega} \subset \mathscr{D}$, there exists a global random attractor in $H_{0}^{2}(D)$ for the random dynamical system associated with system (3.1)-(3.3).

\section{Competing interests}

The authors declare that they have no competing interests.

\section{Authors' contributions}

The authors contributed equally and significantly in writing this paper. All authors read and approved the final manuscript.

\section{Author details}

'Department of Mathematics, China University of Mining and Technology Beijing, Beijing, 100083, China. ${ }^{2}$ Department of Information and Computation Science, Guangxi University of Science and of Technology, Guangxi, 545006, China.

\section{Acknowledgements}

This work was supported by the National Natural Science Foundation of China (Grant No. 11371183) and Guangxi Provincial Natural Science Foundation (No. FAA118016).

Received: 17 October 2016 Accepted: 20 January 2017 Published online: 02 February 2017

\footnotetext{
References

1. Swift, JB, Hohenberg, PC: Hydrodynamic fluctuations at the convective instability. Phys. Rev. A 15(1), 319-328 (1977)

2. Hohenberg, PC, Swift, JB: Effects of additive noise at the onset of Rayleigh-Bénard convection. Phys. Rev. A 46, 4773-4785 (1992)

3. Pomeau, Y, Manneville, P: Wave length selection in cellular flows. Phys. Lett. A 75, 296-298 (1980)

4. Lega, J, Moloney, JV, Newell, AC: Swift-Hohenberg equation for lasers. Phys. Rev. Lett. 73, 2978-2981 (1994)

5. Doelman, A, Standstede, B: Propagation of hexagonal patterns near onset. Eur. J. Appl. Math. 14, 85-110 (2003)

6. Hale, JK: Asymptotic Behaviour of Dissipative Systems. AMS, Providence (1988)

7. Sell, G, You, Y: Dynamics of Evolutionary Equations. Springer, New York (2002)
} 
8. Temam, R: Infinite-Dimensional Dynamical Systems in Mechanics and Physics. Springer, New York (1997)

9. Lin, G, Gao, H, Duan, J, Ervin, VJ: Asymptotic dynamical difference between the nonlocal and local Swift-Hohenberg models. J. Math. Phys. 41(4), 2077-2089 (2000)

10. Polat, M: Global attractor for a modified Swift-Hohenberg equation. Comput. Appl. Math. 57, $62-66$ (2009)

11. Song, L, Zhang, Y, Ma, T: Global attractor of a modified Swift-Hohenberg equation in $H^{k}$ spaces. Nonlinear Anal. 72 , 183-191 (2010)

12. Oh, J, et al.: Dynamics of fluctuations in a fluid below the onset of Rayleigh-Bénard convection. Phys. Rev. E 69, $021106(2004)$

13. Rehberg, l, et al.: Thermally induced hydrodynamic fluctuations below the onset of electroconvection. Phys. Rev. Lett. 67, 596-599 (1991)

14. Blömker, D, Hairer, M, Pavliotis, GA: Stochastic Swift-Hohenberg equation near a change of stability. Proceedings of Equa. Diff. 11, 27-37 (2005)

15. Crauel, H, Debussche, A, Flandoli, F: Random attractors. J. Dyn. Differ. Equ. 9, 307-341 (1997)

16. Crauel, H, Flandoli, F: Attractors for random dynamical systems. Probab. Theory Relat. Fields 100, 365-393 (1994)

17. Da Prato, G, Debussche, A, Temam, R: Stochastic Burgers' equation. NoDEA, Nonlinear Differ. Equ. Appl. 1, 389-402 (1994)

18. de Bouard, A, Debussche, A: On the stochastic Korteweg-de Vries equation. J. Funct. Anal. 154, 215-251 (1998)

19. de Bouard, A, Debussche, A: A stochastic nonlinear Schrödinger equation with multiplicative noise. Commun. Math. Phys. 205, 161-181 (1999)

20. Arnold, L: Random Dynamical Systems. Springer, New York (1998)

21. Duan, J, Wang, W: Effective Dynamics of Stochastic Partial Differential Equations. Elsevier, London (2014)

22. Fan, X: Attractors for a damped stochastic wave equation of Sine-Gordon type with sublinear multiplicative noise. Stoch. Anal. Appl. 24, 767-793 (2006)

23. Da Prato, G, Zabczyk, J: Stochastic Equations in Infinite Dimensions. Cambridge University Press, New York (1992)

\section{Submit your manuscript to a SpringerOpen ${ }^{\circ}$ journal and benefit from:}

- Convenient online submission

- Rigorous peer review

Immediate publication on acceptance

- Open access: articles freely available online

- High visibility within the field

- Retaining the copyright to your article 